\title{
Wireless Sensor and Communication Nodes with Energy Harvesting
}

\author{
Mehmet Şafak \\ Hacettepe University, Ankara, Turkey, E-mail: msafak@ee.hacettepe.edu.tr \\ Received September 2013; Accepted November 2013 \\ Publication January 2014
}

\begin{abstract}
Energy is a valuable resource in wireless communication, navigation and sensor nodes. Maintenance and replacement of batteries in battery-driven nodes may not be possible, cost-effective or suitable for many applications. On the other hand, energy harvesting provides sustainable and independent operation with very long life-time but usually with unregulated power flow. The design approaches in present systems based on a regulated flow of power from an energy storage device are not optimal for energy harvesting nodes. This paper briefly reviews the capabilities of the present energy harvesting technologies and some intelligent design approaches based on unregulated supply power to energy harvesting nodes. The paper also presents a brief review of the alternative approaches for in-body communications with energy harvesting. The use of $\mathrm{THz}$ band does not seem to be feasible because of the excessive signal attenuation in the in-body channel. However, radio-frequency identification (RFID) with inductive magnetic coupling looks appropriate due to the fact that the human body behaves like free space to the magnetic field but strongly attenuates the electric field.
\end{abstract}

Keywords: Energy harvesting, wireless sensor networks, wireless communications, green communications, near-field communications, RFID, nanogenerators, $\mathrm{THz}$ communications, biological communication channels.

Journal of Communication, Navigation, Sensing and Services, Vol. 1, 47-66.

doi: 10.13052/jconasense2246-2120.113

(c) 2014 River Publishers. All rights reserved. 


\section{Introduction}

Energy is a valuable resource in communications, navigation, sensing and services (CONASENSE)-related applications. Operation of, for example, wireless communications and sensor networks (WSN) requires a regulated flow of power from the energy/power source to the electronic equipment. If electricity is not available on-site, the systems may be operated by battery or harvesting energy from their close vicinity. However, irrespective of whether the required electric energy is provided by the mains, the battery or energy harvesting, minimization of the consumed energy is strongly desired because of reasons such as cost, equipment life-time and electromagnetic compatibility. In WSN, sensors are usually battery-operated because of the difficulty and/or inconveniance of reaching sensor nodes in remote locations, high cost of maintainence and replacement. Hence, the energy efficiency of wireless sensor nodes determines the life-time of battery-operated sensor nodes, which are required to provide independent, sustainable and continuous operation. The design of new generation systems should take into account of the limitations of energy harvesting, e.g., scarcity, unregulated flow and non-availability of power in some time intervals which can not be predicted beforehand.

As shown in Figure 1, a communication or a sensor node may be considered to be composed of supply and demand sides. The demand side consists of energy consuming units such as a sensor, a signal processing unit, a wireless transceiver and a buffer, either to store the sensed data and/or for the data to be transmitted/received [1]. A sensor node differs from a communication node by the presence of a sensor. Transceivers typically use Bluetooth or Zigbee protocols to communicate within a range of maximum $30 \mathrm{~m}$ and require output power levels in the order of 2-100 $\mathrm{mW}$ (see Table 1) [2]. Hence, power levels needed by a sensor node may be in the order of a few $100 \mathrm{mWs}$. However, the energy consumption in transceivers may be decreased by reducing the data to be transmitted/received, chosing adaptive coding and modulation strategies, using energy efficient transmission scheduling, routing and medium access control as well as exploiting power saving modes (sleep/listen) [3], [4].

The supply side of an energy harvesting node consists of energy storage and energy harvesting systems. Energy harvesting implies the collection of energy from ambient sources and converting it into electrical energy. On the other hand, the lifetime of energy harvesting systems is theoretically infinite. However, battery-operated nodes do not have an energy harvester and their life-time is mainly limited by the battery capacity. 


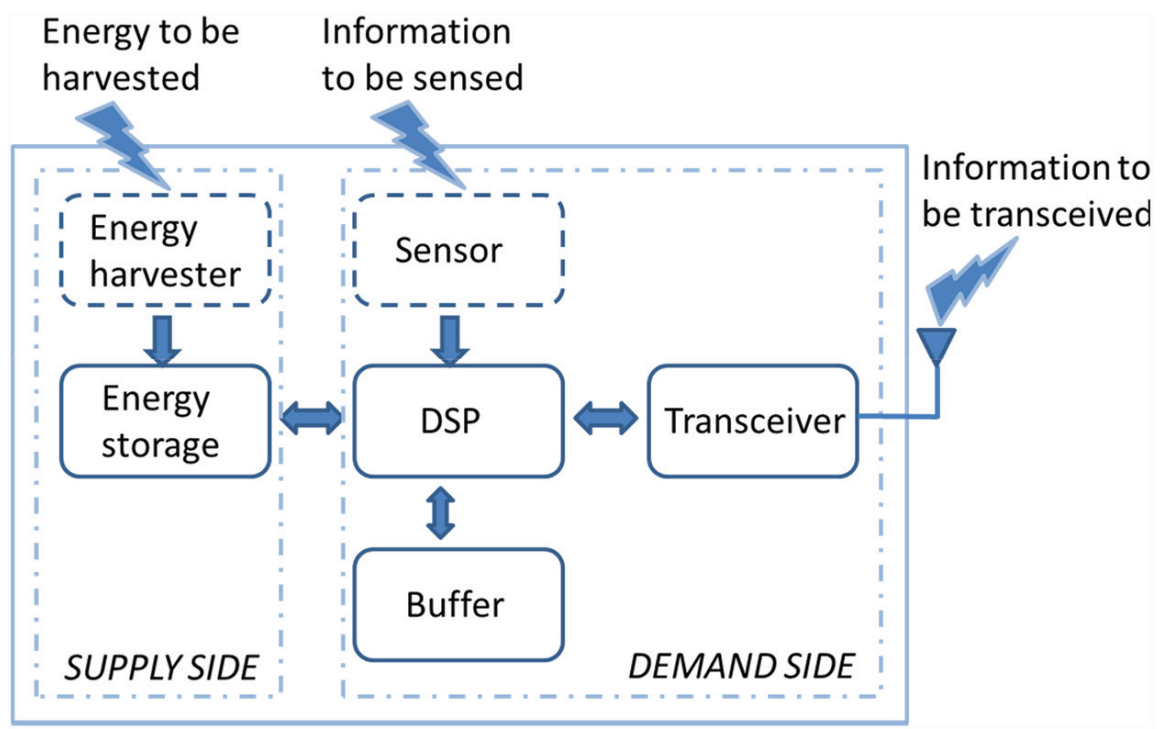

Figure 1 Block diagram of a sensor/communication node from energy perspective [3].

Table 1 Required output power and range of Bluetooth and Zigbee [2].

\begin{tabular}{lllll}
\hline & & Output & \\
Transceiver & Frequency & Bit Rate & Power & Range \\
\hline IEEE 802.15.1 (Bluetooth) & ISM band(2.4 GHz) & $1 \mathrm{Mbps}$ & $100 \mathrm{~mW}$ & $<30 \mathrm{~m}$ \\
IEEE 802.15.4 (Zigbee) & ISM band(2.4 GHz) & $250 \mathrm{kbps}$ & $2 \mathrm{~mW}$ & $<30 \mathrm{~m}$ \\
\hline
\end{tabular}

Recently remarkable improvements are observed in power density (W/kg), efficiency, amount of supplied power and the capacity (Amp.-hour) in the area of energy storage [5]. Nevertheless, operation by batteries still has its limitations and may not be suitable for certain applications. Hence, there is a strong demand for energy harvesting systems which can generate their own energy from their environment. Since energy to be harvested may not always be available and predictable, energy harvesting systems usually employ batteries for storing the harvested energy for present/future use. The harvesting efficiency and the availability of energy source are the fundamental issues to be considered. Since existing node designs are mostly based on the continuous flow and availability of the electric energy, these nodesmay not operate optimally with energy harvesting and novel approaches are required. 
The next section of this paper will briefly review the capabilities offered by battery operation and energy harvesting. Section 3 will provide a brief discussion of intelligent node designs based on energy harvesting. Electromagnetic wave propagation in biological tissues in various frequency bands will be reviewed in Section 4 in order to discover the potential and the limitations of in-body communications. Section 5 will present near-field communications, which is based on wave-propagation in the near-field (Fresnel) region, and its use especially for in-body communications. Section 6 will provide a discussion of the potential, limitations of nanogenerators and their use in nano-sized sensor nodes operating in the $\mathrm{THz}$ band. Finally, the conclusions will be presented in Section 7.

\section{Energy Supply}

Battery-driven systems use stored chemical energy to supply the electrical energy needed by a node. Hence the batteries and the battery-driven systems have a finite lifetime. It is noted that regular maintenance and replacement of batteries may be difficult and costly when nodes are remotely located and/or densely populated. To increase the life time of battery-driven nodes, one may use either higher battery capacity, which implies increased cost, or low-duty cycle operation with lower sensing reliability. Increased transmission range requires higher transmit power levels, while lower transmission range (lowering transmit power) implies more hops and usually higher energy usage at multiple nodes. If both demand and supply sides of a node as shown in Figure 1 are optimized, the energy need may be decreased by an order of magnitude compared to present designs; then, the energy need of the demand side may be reduced to the order of several $10 \mathrm{~mW}$ [6].

Table 2 presents the results of a recent survey of the energy levels that can be supplied by various battery types. One may easily observe from Table 2 that even if the energy demand of a node is decreased by an order of magnitude with intelligent designs, battery-operated systems sustain power levels in the order of several $10 \mathrm{~mW}$ only for limited time durations, hence limited life-time.

Energy harvesting requires both an energy source to be harvested and an energy harvesting sensor, which should be matched to the energy source for optimizing the harvesting process. The energy sources to be harvested may be thermal (including solar energy and human body), mechanical (translational/vibrational), chemical, electromagnetic, wind etc. One may use piezoelectric sensors (vibrational energy), thermocouples (thermal energy), photovoltaic cells (solar energy), wind turbines (wind energy) and 
Wireless Sensor and Communication Nodes with Energy Harvesting

Table 2 Present technology for battery power [5].

\begin{tabular}{|c|c|c|c|c|c|c|}
\hline $\begin{array}{l}\text { Battery } \\
\text { Type }\end{array}$ & $\begin{array}{l}\text { Nominal } \\
\text { Voltage } \\
\text { (V) }\end{array}$ & $\begin{array}{l}\text { Capacity } \\
\text { Capacity }\end{array}$ & $\begin{array}{l}\text { Weight } \\
\text { Energy } \\
\text { Density } \\
\text { (Wh/kg) }\end{array}$ & $\begin{array}{l}\text { Power } \\
\text { Density } \\
(\mathrm{W} / \mathrm{kg})\end{array}$ & $\begin{array}{l}\text { Efficiency } \\
(\%)\end{array}$ & $\begin{array}{l}\text { Self } \\
\text { Discharge } \\
\text { (\%month) }\end{array}$ \\
\hline SLA & 6 & 1300 & 26 & 180 & $70-92$ & 20 \\
\hline $\mathrm{NiCd}$ & 1.2 & 1100 & 42 & 150 & $70-90$ & 10 \\
\hline NiMH & 1.2 & 2500 & 100 & $250-1000$ & 66 & 20 \\
\hline Li-ion & 3.7 & 740 & 165 & 1800 & 99.9 & $<10$ \\
\hline $\begin{array}{l}\mathrm{Li}- \\
\text { polymer }\end{array}$ & 3.7 & 930 & 156 & 3000 & 99.8 & $<10$ \\
\hline
\end{tabular}

radio-frequency (RF) systems (electromagnetic energy) to harvest various energy types.

Limitations in energy harvesting usually come from uncontrollability and unpredictability of the energy source and the efficiency with which the harvested energy is converted into electrical energy. Consequently, the level of the harvested energy and its management (harvesting architecture) is critical in the system operation. In that context, storing or no-storing of the harvested energy and the optimal design of the demand side are serious issues to be considered. Energy usage should be carefully coordinated and optimized between supply and demand sides, including signal processing and communication architectures, sleep scheduling, energy-efficient communication protocols and adaptive coding and modulation [3].

$R F$ energy harvesting is mainly based on magnetic inductive coupling due to the Faraday's law, which also provides the basic principles of electric motors and generators. A loop carrying a (sufficiently-fast) time-varying current, creates a magnetic field around itself. This induces an open-circuit voltage around the terminals of a receive loop, when it is brought to the vicinity of the transmit loop. The open-circuit voltage is proportional to the magnetic flux through the receive loop. In addition to the frequency and the amplitude of the transmit loop current, the dimensions, relative directions, the distance between and the number of turns of transmit and receive loops determine the flux and hence the open-circuit voltage. The two loops are required to be in the Fresnel region of each other, where radiative near-field communications takes place. The voltage induced at the terminals of the receive loop may be used either for responding the request of the transmitting loop as in RFID systems, or for charging a battery connected to its terminals. Induced voltage levels in these systems are reported to be approximately $0.5 \mathrm{~V}$ [5]. 
Vibrational energy harvesters, made of piezoelectric material, convert vibrational (mechanical) energy into electrical energy. An electric potential is induced at the terminals of a piezoelectric material if it undergoes a strain (e. g., due to body motion, muscle stretching, breathing, sonic waves) due to the polarization of ions in the crystal. This electric potential may drive a transient flow of electrons in the external circuit, hence it generates electric energy; this is the fundamental principle of the nanogenerator. It can as well cause the flow of charge carriers through a semiconductor material, resulting in piezopotential-gated field-effect-transistors, diodes and sensors; this is the so-called principle of piezotronics [7] [8]. Piezoelectric energy harvesters directly convert a mechanical vibration into a relatively high voltage $(\sim 1-$ $20 \mathrm{~V})$ and output current $(\sim 1-100 \mu \mathrm{A})$ with a duty cycle less than $3 \%$ in RF transmissions. Piezoelectric energy harvesters are used in railway and road tunnels to produce electricity by using the vibrations induced by trains and cars. The applications also include shoe-powered RF tag system, which converts the vibrational energy created by walking subjects, and self-powered door bells, which produce electrical energy using the vibrations when a door bell is pressed [9]. Electrical power may also be generated for applications in bio-microelectromechanical systems (MEMS) and microelectronic systems by inserting piezoelectric generators between the coping of a dental crown and the metal abutment [10].

Thermal energy (TE) generators convert temperature differences into electrical energy based on the so-called Seebeck effect which was first discovered in 1821 by T. J. Seebeck. A simple thermal energy generator may be made by heating one face of a TE module and cooling the other face, causing an electric current flowing through a load connected to its terminals. A TE generator has usually a long life cycle, no moving parts, simple and high reliability but lowefficiency (5-10\%). Among the thermal energy sources, one may list the waste heat in industrial applications and solar thermal energy. Thermal energy can also be harvested from a human body exploiting small temperature gradients. Seiko thermic watch harvests $22 \mu \mathrm{W}$ from a human body and uses this power to drive the wrist watch and charges a $4.5 \mathrm{mAh}$ lithium-ion battery. When it is worn on the wrist, the watch uses the body heat absorbed from its back case to generate power with its thermal converter. The power generating capability is a function of the difference between the air temperature and the body surface temperature. If the air temperature is equal to, or greater than, the surface temperature of the body, the watch is unable to generate power [11]. Human body has potential in harvesting thermal and kinetic energies. The harvested power depends on whether the subject is walking or running. For example, it 
Table 3 Energy sources and their energy harvesting potential.

\begin{tabular}{llll}
\hline $\begin{array}{l}\text { Energy } \\
\text { Source }\end{array}$ & $\begin{array}{l}\text { Available } \\
\text { Power }\end{array}$ & $\begin{array}{l}\text { Conversion } \\
\text { Efficiency }\end{array}$ & $\begin{array}{l}\text { Harvested } \\
\text { Power }\end{array}$ \\
\hline Solar [4] & $100 \mathrm{~mW} / \mathrm{cm}^{2}$ & $15 \%$ & $15 \mathrm{~mW} / \mathrm{cm}^{2}$ \\
Wind [4] & - & - & $120 \mathrm{mWh} /$ day \\
Finger motion [4] & $19 \mathrm{~mW}$ & $11 \%$ & $2.1 \mathrm{~mW}$ \\
Footfalls [4] & $67 \mathrm{~W}$ & $7.5 \%$ & $5 \mathrm{~W}$ \\
Exhalation [4] & $1 \mathrm{~W}$ & $40 \%$ & $0.4 \mathrm{~W}$ \\
Breathing [4] & $0.83 \mathrm{~W}$ & $50 \%$ & $0.42 \mathrm{~W}$ \\
Blood pressure [4] & $0.93 \mathrm{~W}$ & $40 \%$ & $0.37 \mathrm{~W}$ \\
Thermoelectric & - & - & $3.5 \mathrm{~mW} / \mathrm{cm}^{2}$ \\
generator [14] & & & $930^{0} \mathrm{C}$ gradient $^{3}$ \\
Human body & - & - & $6 \mu \mathrm{W} / \mathrm{cm}^{3}($ thermal) \\
(walking & & & $1.3 \mu \mathrm{W} / \mathrm{cm}^{3}(\mathrm{kinetic})$ \\
subject) [10] & & $73 \% @ 5 \mathrm{~cm}$ & - \\
Inductive magnetic & - & $24 \% @ 15 \mathrm{~cm}$ & \\
coupling [15] & & & \\
\hline
\end{tabular}

is reported in [12] that one can harvest approximately $6 \mu \mathrm{W}$ thermal energy and a kinetic energy of $1.3 \mu \mathrm{W} / \mathrm{cm}^{3}$ from the body of a walking subject. The corresponding numbers are $10 \mu \mathrm{W}$ and $\sim 35 \mu \mathrm{W}$ for a running subject.

The human body may be used in various other ways to harvest energy, e.g., via blood pressure and breath, which are uncontrollable by the user, and via finger motion, paddling (bycle dynamo) and walking (shoes), which are user controllable. Wearable bio-sensors such as gloves, wrist-watches, rings, patches, earlobes, intelligent clothes, eye-glasses, accelerometers, glucose monitor, electrocardiograph, pulse sensor, oxygen-level monitor, temperature sensor, respiratory meter can potentially be used for harvesting energy from human body [13].

Table 3 shows a summary of some energy sources, their characteristics, required energy harvesting technology and the amount of energy that can potentially be harvested.

\section{Intelligent Designs for Energy Harvesting Nodes}

Classical design of sensor/communication nodes is based on the availability of a continuous flow of a constant power level (infinite energy) to the demand side. On the other hand, for the self-powered nodes to be practical, dramatic reductions are strongly desired in the dissipated power levels since energy harvesting technology is presently far from satisfying present needs. Energy sources may be (un)controllable and/or (un)predictable for 
energy harvesting; solar energy is predictable but uncontrollable, while RF energy harvesting in an RFID system may be controllable and predictable at the same time. Therefore, limited power that can be harvested sets a constraint on the average power consumed by the demand side for selfpowered operation. This implies that energy harvesting, storing and processing technologies should be improved so as to help sustainable and continuous operation.

Even if infinite energy becomes available to the supply side, energy generation may not be continuous and/or rate of generation may be limited. Storing the harvested energy may partially alleviate this problem since it may regulate the power flow. Nevertheless, electronic devices with classical design can not reliably operate under these conditions. Therefore, energy generation profile of the supply side should be matched to the energy consumption profile of the demand side. This requires a system-level approach involving variation-tolerant architectures, ultra-low voltage levels and highly digital RF circuits. In addition, one needs DSP architecture and circuits which are energy-efficient, energy-scalable, and robust to variations in the output voltage/power levels of the supply side. Energy-scalable hardware may call for techniques for approximate processing, which implies a trade-off between power and arithmetic precision [16]. In wireless sensor networks, the demand side may be designed with sleep/awake periods in synchronism with energy harvesting by the supply side. Energy consumption policy may be optimized in seeking a tradeoff between the throughput and the life-time of the sensor node [17]. Such approaches are believed to result in more than an order of magnitude energy reduction compared to present systems [6].

In some projects like PicoRadio (Berkeley), $\mu$ AMPS (MIT), WSSN (ICT Vienna) and GAP4S (UT Dallas), densely populated low-cost sensor nodes are foreseen to operate with power levels of approximately $100 \mu \mathrm{W}$; such power level is believed to be within the capabilities of energy harvesting [18]. Even though dramatic improvements are still needed in the energy harvesting technologies for self-operated nodes, rapidly-evolving energy harvesting technologies are believed to be promising.

\section{Electromagnetic Wave Propagation in Biological Tissues}

An electric field propagating in a lossy dielectric medium may be written as

$$
E=E_{0} e^{-\gamma r}=E_{0} e^{-\alpha r} e^{-j \beta r}
$$


where $\gamma=\alpha+j \beta$ denotes the complex propagation constant, $r$ is the distance and $E_{0}$ is the value of the electric field at $r=0$. In a lossy dielectric medium, the complex propagation constant may be written as [19]

$$
\begin{aligned}
& \gamma=\alpha+j \beta=k_{0} \sqrt{\varepsilon_{r}-j p} \\
& \alpha=k_{0} \sqrt{\frac{\sqrt{\varepsilon_{r}^{2}+p^{2}}-\varepsilon_{r}}{2}} \\
& \beta=k_{0} \sqrt{\frac{\sqrt{\varepsilon_{r}^{2}+p^{2}}+\varepsilon_{r}}{2}} \\
& p=\frac{\sigma}{w \varepsilon_{0}}=\frac{18 \sigma}{f_{G H z}}
\end{aligned}
$$

where $w$ and $k_{0}$ denote respectively the radial frequency and the free-space wave-number. The relative dielectric constant (permittivity) $\epsilon_{r}$ and the conductivity of the lossy dielectric medium $\sigma$ are both assumed to be independent of frequency. Note that, in a non-conducting medium $(\sigma=0)$, the attenuation constant $\alpha$ vanishes and $\beta=k_{0} \sqrt{\varepsilon_{r}}$, as expected. In a lossy dielectric medium, $\sigma$ is related to the dispacement current. The signal attenuation in $\mathrm{dB}$ at a distance $r$ is given by

$$
20 \log \left(e^{-\alpha r}\right)=8.68 \alpha r \quad(d B)
$$

where $8.68 \alpha(\mathrm{dB} / \mathrm{m})$ denotes the attenuation coefficient.

In biological tissues, the dielectric constant is a frequency- and temperature-dependent complex quantity and provides a measure of the interaction of electromagnetic waves with tissue constituents at cellular and molecular levels [20], [21]. There is a lag (delay) between changes in polarization in the tissue and time-changes in the applied electric field due to the relaxation process. Consequently, the permittivity of a dielectric material becomes a complicated, complex-valued function of the frequency, which implies frequency-dependent and delayed response of live tissues to wave propagation. This dispersion effect is analogous to hysteresis in changing magnetic fields.

Frequency dependence of the relative dielectric constant may be modeled by the Cole-Cole equation:

$$
\varepsilon_{r}=\varepsilon_{\infty}+\frac{\varepsilon_{s}-\varepsilon_{\infty}}{1+(j w \tau)^{1-\alpha}}
$$

which reduces to the so-called Debye equation for $\alpha=0$. Here, $\epsilon_{s}$ and $\epsilon_{\infty}$ denote respectively the values of the relative dielectric constant for $w \tau<<1$ (low-frequencies) and $w \tau>>1$ (high frequencies). The static value $\epsilon_{s}$ of the 
relative dielectric constant is proportional to the water content of the tissue. The value of $\alpha$ is equal to zero for pure water and negligibly small for body fluids but greater than zero for most tissues. Here, $\tau$ denotes the mean relaxation time, which is longer than that for pure water.

An approximation to the complex dielectric constant given by (4) is given by [22]

$$
\varepsilon_{r} \cong \varepsilon_{\infty}+\frac{\beta_{0}+j w \beta_{1}}{\alpha_{0}-w^{2}+j w \alpha_{1}}
$$

where $\alpha_{0}, \beta_{0}, \alpha_{1}, \beta_{1}$ are fitting parameters to the measured data. Inserting the value of the complex relative dielectric constant given by (4) or (5) into (2), one can easily find the corresponding values of the coefficients, $\alpha$ and $\beta$, of the complex propagation constant.

The relative magnetic permeability of a biological tissue in the RF band of interest is equal to unity $\left(\mu_{r}=1\right)$. Therefore, biological tissues may be characterized electrically by the relative dielectric constant and the conductivity only. A comprehensive experimental study [20] provides in-vivo measurement values of these two parameters of some animal tissues at frequencies varying between $50 \mathrm{MHz}$ and $20 \mathrm{GHz}$. Some results for human tissues are also given. The provided data may be used for modeling the in-body communication channel, e.g., for signal transmission between implant-devices and devices on/outside the body.

Table 4 shows the measurement results for relative dielectric constant and conductivity of some in-vivo body tissues of $\sim 50 \mathrm{~kg}$ pigs at $50 \mathrm{MHz}, 1 \mathrm{GHz}$ and $20 \mathrm{GHz}$. The corresponding attenuation coefficients in $\mathrm{dB} / \mathrm{cm}$ are also provided. Table 4 clearly shows that, for electromagnetic wave propagation in biological tissues, the attenuation coefficient expressed in $\mathrm{dB} / \mathrm{cm}$ is acceptably low at $50 \mathrm{MHz}$ but increases to relatively high values at $1 \mathrm{GHz}$ and becomes excessively high at $20 \mathrm{GHz}$. On the other hand, in view of the typical transmission ranges in human body, far-field communications is not possible at $50 \mathrm{MHz}$ (the wavelenth $\lambda=6 \mathrm{~m}$ ) since transmit and receive antennas may not be in the far-fields of each other. At $1 \mathrm{GHz}(\lambda=0.3 \mathrm{~m})$, although the transmitter and receiver may be located in far-field regions of each other, very high transmit powers are required for compensating excessively high channel attenuations. Such high transmit powers are also unacceptable for health reasons.

In addition to the very high attenuation coefficients for in-body communications, as given by Table 4, one should also consider the freespace path loss, $(4 \pi r / \lambda)^{2}$, which is proportional to $r^{2}$ and $f^{2}$. Also note that 
Table 4 Relative permittivity, conductivity and attenuation coefficient of biological tissues at $50 \mathrm{MHz}, 1 \mathrm{GHz}$ and $100 \mathrm{GHz}$ (based on [20]*).

\begin{tabular}{|c|c|c|c|c|}
\hline Tissue & Parameter & $50 \mathrm{MHz}$ & $1 \mathrm{GHz}$ & $20 \mathrm{GHz}$ \\
\hline \multirow[t]{3}{*}{ Liver } & $\epsilon_{r}$ & 96 & 50 & 30 \\
\hline & $\sigma(\mathrm{S} / \mathrm{m})$ & 0.7 & 1 & 20 \\
\hline & $8.68 \alpha(\mathrm{dB} / \mathrm{cm})$ & 0.85 & 2.28 & 57.41 \\
\hline \multirow[t]{3}{*}{ Lung (deflated) } & $\epsilon_{r}$ & 87 & 40 & 26 \\
\hline & $\sigma(\mathrm{S} / \mathrm{m})$ & 0.8 & 1 & 17 \\
\hline & $8.68 \alpha(\mathrm{dB} / \mathrm{cm})$ & 0.94 & 2.53 & 52.49 \\
\hline \multirow[t]{3}{*}{ Long bone } & $\epsilon_{r}$ & 43 & 25 & 15 \\
\hline & $\sigma(\mathrm{S} / \mathrm{m})$ & 0.2 & 0.4 & 8 \\
\hline & $8.68 \alpha(\mathrm{dB} / \mathrm{cm})$ & 0.41 & 1.3 & 32.9 \\
\hline \multirow{3}{*}{$\begin{array}{l}\text { Bone marrow ( } 50 \% \\
\text { in-vivo) }\end{array}$} & $\epsilon_{r}$ & 67 & 35 & 22 \\
\hline & $\sigma(\mathrm{S} / \mathrm{m})$ & 0.6 & 0.8 & 14 \\
\hline & $8.68 \alpha(\mathrm{dB} / \mathrm{cm})$ & 0.81 & 2.17 & 47.1 \\
\hline \multirow[t]{3}{*}{ Fat (infiltrated) } & $\epsilon_{r}$ & 16.5 & 15.5 & 9.0 \\
\hline & $\sigma(\mathrm{S} / \mathrm{m})$ & 0.25 & 0.4 & 6.0 \\
\hline & $8.68 \alpha(\mathrm{dB} / \mathrm{cm})$ & 0.56 & 1.62 & 31.44 \\
\hline \multirow[t]{3}{*}{ Skin (in-vivo) } & $\epsilon_{r}$ & 90 & 38 & 22 \\
\hline & $\sigma(\mathrm{S} / \mathrm{m})$ & 0.2 & 0.6 & 13 \\
\hline & $8.68 \alpha(\mathrm{dB} / \mathrm{cm})$ & 0.32 & 1.58 & 43.9 \\
\hline \multirow[t]{3}{*}{ Vitreous humour } & $\epsilon_{r}$ & 105 & 69 & 40 \\
\hline & $\sigma(\mathrm{S} / \mathrm{m})$ & 1.8 & 1.9 & 60 \\
\hline & $8.68 \alpha(\mathrm{dB} / \mathrm{cm})$ & 1.5 & 3.64 & 134.1 \\
\hline \multirow{3}{*}{$\begin{array}{l}\text { Cerebrospinal fluid } \\
\text { (CSF) }\end{array}$} & $\epsilon_{r}$ & 105 & 70 & 44 \\
\hline & $\sigma(\mathrm{S} / \mathrm{m})$ & 1.8 & 1.9 & 60 \\
\hline & $8.68 \alpha(\mathrm{dB} / \mathrm{cm})$ & 1.51 & 3.62 & 130.2 \\
\hline
\end{tabular}

*Tabulated values are obtained by reading the curves provided in [20] and hence may contain some reading errors.

multi-path propagation, shadowing and scattering effects due to inhomogeneities in the electrical parameters of the tissues make the in-body communications even more challenging.

\section{Near-Field Communications}

Near-field communications refers to communications in the Fresnel region, defined by the range $0.62 \sqrt{D^{3} / \lambda}<r<2 D^{2} / \lambda$, where $D$ denotes the largest antenna dimension. In this region, the relation between electric and magnetic fields radiated by an antenna is not as simple as in the far-field (Fraunhofer) region, which is defined by $r>2 D^{2} / \lambda$. In the Fresnel region, 
electric and magnetic fields do not have plane wavefronts and the ratio of electric to magnetic field intensities is not equal to the free-space intrinsic impedance, $120 \pi \mathrm{Ohm}$, as in the far-field region. Nevertheless, the radiative field components still dominate the reactive field components and are used for near-field communications. Note that near- and far-field distances defined above are meaningful for antenna sizes exceeding the wavelength $(D>\lambda)$. For electrically small antennas with $D<\lambda / 2$, the Fresnel region is usually assumed to be bounded by the range $\lambda /(2 \pi)<r<\lambda$ [23].

The efficiency of near-field communications is critically dependent on coupling between transmit and receive antennas which are located in each other's Fresnel regions. Depending on the frequency of operation, the propagation medium and the types of antennas used, it might be more appropriate to use electric or magnetic coupling between antennas. The electrical characteristics (permittivity, magnetic permeability and conductivity) of the medium between transmit and receive antennas also plays a critical role in choosing the type of coupling. For example, a near-field communication system between, for example, implant devices inside a human body and an antenna over the skin should prefer magnetic coupling since the propagation medium (human body) is characterized by high conductivity and high relative dielectric constants (see Table 4). Since electric field suffers much higher attenuations in the in-body channel, magnetic coupling between transmit and receive antennas is more appropriate than electrical coupling. Since $\mu_{r}=1$ in the human body, magnetic fields do not suffer losses in addition to the free-space loss.

RFID systems constitute one of the most notable examples of inductive magnetic coupling which is commonly used in near-field communications [24]. The magnetic field radiated by time-varying currents in the RFID reader loop antenna induces a voltage around the terminals of the loop antenna of the RFID tag. The induced voltage is used for transmitting the information requested by the RFID reader. The efficiency of coupling between transmitter and receiver, hence the induced voltage, is determined by the fraction of the magnetic flux created by the transmit loop antenna coupled into the receive (RFID tag) loop antenna. Near-field coupling between antennas is highly dependent on the types, electrical sizes, radiation patterns, relative orientations and impedance matching of the antennas employed and the distance between them [25].

The same principle also applies for distant charging of a battery connected at the terminals of the receive loop antenna. For example, batteries of implant devices may be charged by inductive magnetic coupling. This also provides an 
example of RF energy harvesting, since received RF energy is converted into chemical energy in an in-body battery. However, it is reported that inductive charging efficiency is lower (hence slower charging) and resistive heating is higher [15]. RF wireless charging of multiple sensors is also considered for smart grid applications by using mobile chargers [26].

A recent study on wireless power transfer, based on strongly coupled magnetic resonance in the near-field, reports coupling coefficients as high as $0.7-0.9$ between primary and secondary loops, as compared to coupling coefficients on the order of 0.1 for inductive magnetic coupling. The distance between the two resonators may be larger than the characteristic sizes of each resonator and, unlike for conventional inductive coupling, energy dissipations are reported to be small [15].

\section{Nanogenerators and Communications in THz Band}

Recently there has been considerable interest in nano-sized sensing and communicating devices that can detect and measure events at nanoscale where energy consumption is believed to be low. Energy harvesting at nanoscale is believed to provide independent, sustainable, maintenance-free, continuous operation. Nanogenerators are foreseen to be used for a variety of applications including intra-body drug delivery, health monitoring, medical imaging, environmental research (air pollution control), military applications (surveillance networks against nuclear, biological, and chemical attacks at nanoscale, and home security), and very high data rate communications [27].

For example, in contrast with today's cancer drugs which kill healthy cells as well as the cancerous ones, drugs may be delivered locally to diseased cells and tumors by miniature bio-MEMS systems that navigate the circulatory system of the human body. However, these machines, which are difficult to recharge within the body, may potentially harvest mechanical energy from the blood cells [10]. Today's technology may not be mature enough to build a motor and power source small enough to squeeze inside the capilleries that feed the tumor. However, magnetic drug carriers as small as $50 \mu \mathrm{m}$ can be steered by external magnetic resonance imaging (MRI) machines [28]. In 2011 it is demonstrated that a gentle straining can yield $1-3 \mathrm{~V}$ with an instantaneous power of $\sim 2 \mu \mathrm{W}$ from an integrated nanogenerator sheet of $1 \mathrm{~cm}^{2}$ using a self-powered nanosensor. Potential applications for MEMS may require power levels in the range $\mu \mathrm{W}$ to $\mathrm{mW}$. Future of nanotechnology research is likely to focus on technologies that provide higher harvested power levels and integration of nanosensors into nanosystems acting 
like living species with sensing, communicating, controlling, navigating and reacting [29].

Communication between sensor nodes is considered to be achievable in the terahertz (THz) band, $0.3-3 \mathrm{THz}(1 \mathrm{~mm} \geq \lambda \geq 0.1 \mathrm{~mm})$. The radiation in the $\mathrm{THz}$ band is non-ionizing and covers part of the infrared (IR) band of the solar spectrum. THz band is currently used and/or considered for use in applications including radioastronomy, space-remote sensing, through-the-wall imaging, and medical surface imaging (for skin cancer).

As for communications in the $\mathrm{THz}$ band, the following issues need to be taken into account. Atmospheric absorption exceeds $100 \mathrm{~dB} / \mathrm{km}$ and rain attenuation exceeds $3 \mathrm{~dB} / \mathrm{km}$ and $10 \mathrm{~dB} / \mathrm{km}$ for rain rates of $5 \mathrm{~mm} / \mathrm{hr}$ and $25 \mathrm{~mm} / \mathrm{hr}$, respectively, at $1 \mathrm{THz}$. On the other hand, free-space propagation loss is equal to $152.4 \mathrm{~dB}$ at a distance of $1 \mathrm{~km}$ at $1 \mathrm{THz}$. This limits the applicability of communications in the $\mathrm{THz}$ band only to short ranges. Since far-field distance is on the order of a fraction of a millimeter, near-field communications is not possible in the THz band. It is noted that, in view of Table 4, for communications between nano-sensors inside a human body and a receiver on-body, the communications budget is not better-off because of the excessively large attenuation. For example, electrical parameters of a deflated lung at $20 \mathrm{GHz}$ are measured to be $\epsilon_{r}=26, \sigma=17 \mathrm{~S} / \mathrm{m}$ (see Table 4); the corresponding attenuation constant is $52.49 \mathrm{~dB} / \mathrm{cm}$. Assuming that these values of the electrical parameters at $20 \mathrm{GHz}$ are also valid at $100 \mathrm{GHz}$, which looks to be a reasonable assumption [19], the attenuation coefficient is found using (3) to be $8.68 \alpha=54.45 \mathrm{~dB} / \mathrm{cm}$ at $100 \mathrm{GHz}$. Hence, the total attenuation at a typical distance of $15 \mathrm{~cm}$ becomes $828.9 \mathrm{~dB}$ including the free-space propagation loss of $55.96 \mathrm{~dB}$. In contrast with this, the attenuation through $15 \mathrm{~cm}$ thickness of biological tissue is measured to be $47 \mathrm{~dB}$ and $39 \mathrm{~dB}$ at $13.5 \mathrm{MHz}$ and $35 \mathrm{MHz}$, respectively, in an inductive coupling near-field communication system [30]. Multipath propagation, shadowing and scattering due to inhomogeneities in the human body may render in-body communication seven more difficult. Transmit power levels required to compensate for such large losses are unlikely to be obtained by energy harvesting. On the other hand, the use of electrically large antennas will lead to problems due to pointing errors.

In summary, due to the unavailability of $\mathrm{THz}$ sources and of compact, solid-state, room-temperature transceivers, we do not foresee $\mathrm{THz}$ commmunications as a good option in near-to mid-terms [31]. However, nanogenerators and nanopiezotronics, which refers to the technology based on coupling piezoelectric and electronics properties, are listed among the top 10 emerging technologies for the future [32]-[34]. 


\section{Conclusions}

With ever-increasing needs for mobility and higher data rates, energy becomes a limiting factor for the performance, the sustainability and the life-time of wireless communication, navigation and sensor nodes. On the other hand, maintenance and replacement of batteries in battery-driven nodes may not be possible, cost-effective or appropriate in many applications. Energy harvesting may provide sustainable and independent operation with very long life-times and is well-suited for some applications such as sensing. However, this technology, presently in its infancy, can not yet meet the requirements for regulated power/energy levels. Present design approaches for nodes in CONASENSE applications, which are mostly based on the supply of regulated power levels from energy storing devices, are therefore not optimal for energy harvesting purposes. In view of limited and noncontinuous availability of the energy to be harvested at varying generation rates, the design of the demand (energy consuming) side of a node should be matched to the supply side, which consists of energy harvesting and storing units. Efficient node designs should therefore be based on low-power consumption and energy-scalability. For in-body communications with energy harvesting, near-field communications with inductive magnetic coupling seem to be appropriate because the human body behaves like free space to the magnetic field but strongly attenuates the electric field. Micro- and nano-systems may be promising for energy harvesting applications in mid- to far-terms. However, in-body communications in the $\mathrm{THz}$ band using micro- and nano-systems seems to be unlikely, mainly because of the excessive signal attenuation in the in-body channel.

\section{References}

[1] Niyato, D., E. Hossain, M. M. Rashid and V. K. Bhargava, "Wireless sensor networks with energy harvesting technologies: a game-theoretic approach to optimal energy management," IEEE Wireless Communications, August 2007, pp. 90-96.

[2] Nakajima, N., Short-range wireless network and wearable bio-sensors for healthcare applications, 2nd Int. Symposium on Applied Sciences in Biomedical and Communication Technologies (ISABEL 2009), 2009, pp.1-6

[3] V. Sharma, U. Mukherji, V. Joseph and S. Gupta, "Optimal energy management policies for energy harvesting sensor nodes," IEEE Trans. Wireless Communications, vol. 9, no.4, pp.1326-1336, April 2010. 
[4] Joseph, V., V. Sharma, and U. Mukherji, "Optimal sleep-wake policies for an energy harvesting sensor node," IEEE ICC, 2009.

[5] Sudevalayam, S., and P. Kulkarni, "Energy Harvesting Sensor Nodes: Surveys and Implications," IEEE Communications Surveys \& Tutorials, vol.13, no.3, 3rd Quarter 2011.

[6] Chandrakasan, A. P., D. C. Daly, J. Kwong and Y. K. Ramadass, "Nextgeneration micro-power systems," IEEE Symposium on VLSI circuits, Digest of technical papers, 2008, pp.2-5.

[7] Wang, Z. L., "Top emerging technologies for self-powered nanosystems: nanogenerators and nanopiezotronics," 3rd Int. Nanoelectronics Conf. (INEC), pp.63-64, 2010.

[8] Chao, P. C.-P., "Energy harvesting electronics for vibratory devices in self-powered sensors, IEEE Sensors Journal, vol. 11, no.12, pp.3106-3121, December 2011.

[9] Kroener, M., "Energy harvesting technologies: energy sources, generators and management for wireless autonomous applications," 9th Int. Multi-Conf. Systems, Signals and Devices (SSD), 2012, pp.1-4.

[10] Mhetre, M. R., N. S. Nagdeo, and H. K. Abhyankar, "Micro energy harvesting for biomedical applications: a review," $3 r d$ Int. Conf. Electronics Computer Technology (ICECT), 2011, vol.3, pp.1-5.

[11] Lu, X., and S.-H. Yang, "Thermal energy harvesting for WSNs,"IEEE Int. Conf. Systems, Man \& Cybernatics (SMC), 2010, pp.3045-3052.

[12] Mitcheson, P. D.,"Energy harvesting for human wearable and implantable bio-sensors," Annual Int. Conf. IEEE Eng. Medicine and Biology Society (EMBS), 2010, pp.3432-3436.

[13] Teng, X. F., Y. T. Zhang, C. C. Y. Poon, and P. Bonato, "Wearable medical systems for p-health, "IEEE Reviews in Biomedical Engineering, vol.1, 2008, pp. 62-74.

[14] Wan, Z. G., Y. K. Tan, and C. Yuen, "Review on energy harvesting and energy management for sustainable wireless sensor networks," IEEE $13^{\text {th }}$ Int. Conf. on Communication Technologies (ICCT), pp. 362-367, 2011.

[15] R. Amirtharajah, J. Collier, J. Siebert, B. Zhou, and A. Chandrakasan, "DSPs for energy harvesting sensors, Applications and Architectures," IEEE Pervasive Computing, July-Sept. 2005, pp. 72-79.

[16] Rajesh, R., V. Sharma and P. Viswanath, "Capacity of fading Gaussian channel with an energy harvesting sensor node," IEEE Globecom Conf. 2011 . 
[17] Tacca, M., P. Monti and A. Fumagalli, "Cooperative and reliable ARQ protocols for energy harvesting wireless sensor nodes," IEEE Trans. Wireless Communications, vol.6, no.7, pp. 2519-2529, July 2007.

[18] Ames, L. A., J. T. de Bettencourt, J. W. Frazier and A. S. Orange, "Radio Communications via Rock Strata," IEEE Trans. Communication Systems, 1963, pp.159-169.

[19] Peyman, A., S. Holden and C. Gabriel, "Dielectric properties of tissues at microwave frequencies," Report, Mobile Telecommunications and Health Research Programme, December 2009.http://www.mthr.org.uk /research_projects/documents/Rum3FinalReport.pdf.

[20] Gabriel, C., S. Gabriel, and E. Corthout, "The dielectric properties of biological tissues: I. Literature survey," Phys. Med. Biol., vol.41, 1996, pp.2231-2249.

[21] Khaleghi, A., I. Balasingham, and R. Chavez-Santiago, "Computational study of the ultra-wideband wave propagation into the human chest," IET Microwaves, Antennas and Propagation, vol. 5, no. 5, pp.559-567, 2011.

[22] Balanis, C. A., Antenna Theory: Analysis and Design (3rd ed.),NJ: J. Wiley, 2005.

[23] Ortiz, S., Jr., "Is near-field communication close to success?," Computer, March 2006, pp.18-20

[24] Chen, Y. S., S.-Y. Chen and H.-J. Li, "Analysis of antenna coupling in near-field communication systems," IEEE Trans. Antennas Propagation, vol.58, no.10, pp.3327-3335, October 2010.

[25] Ho, S. L., J. Wang, W. N. Fu, and M. Sun, "A comparative study between novel witricity and traditional inductive magnetic coupling in wireless charging," IEEE Trans. Magnetics, vol.47, no.5, pp.1522-1525, May 2011.

[26] M. E. Kantarci, and H. T. Mouftah, "SuReSense: sustainable wireless rechargeable sensor networks for the smart grid," IEEE Wireless Communications, pp. 30-36, June 2012.

[27] Jornet, J. M., and I. F. Akyildiz, "Joint energy harvesting and communication analysis for perpetual wireless nanosensor networks in the Terahertz band," IEEE Trans. Nanotechnology, vol.11, no.3, pp.570-580, May 2012.

[28] Martel, S., "Journey to the center of a tumor," IEEE Spectrum, pp. 49-53, October 2012. 
[29] Wang, Z. L.,"Nanogenerators for self-powering nanosystems and piezotronics for smart MEMS/NEMS," IEEE 24th Int. Conf. MEMS, pp. 115-120, 2011.

[30] T. Yamada, et al., "Battery-less wireless communication system through human body for in-vivo healthcare chip," 2004 Topical Meeting on Silicon Monolithic Integrated Circuits in RF Systems, pp.322-325.

[31] Armstrong, C. M., "The truth about terahertz,"IEEE Spectrum, pp.28-33, Sept. 2012.

[32] Wang, Z. L., Top emerging technologies for self-powered nanosystems: nanogenerators and nanopiezotronics, 3rd Int. Nanoelectronics Conf. (INEC), 2010, pp.63-64.]

[33] New Scientists (Top 10 Future Technologies) http://www.newscientist. com/article/mg20126921.800-ten-scifi-devices-that-could-soon-be-in-y our-hands.html?full=true] MIT Technology Review (Top 10 Emerging Technology in 2009), http://www.technologyreview.com/video/ ?vid $=257$

\section{Biography}

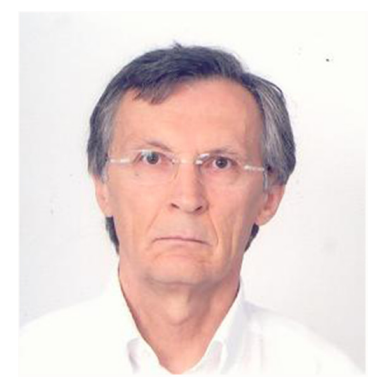

Mehmet Şafak received the B.Sc. degree in Electrical Engineering from Middle East Technical University, Ankara, Turkey in 1970 and M.Sc. and Ph.D. degrees from Louvain University, Belgium in 1972 and 1975, respectively.

He joined the Department of Electrical and Electronics Engineering of Hacettepe University, Ankara, Turkey in 1975. He was a postdoctoral research fellow in Eindhoven University of Technology, The Netherlands during the academic year 1975-1976. From 1984 to 1992, he was with the Satellite Communications Division of NATO C3 Agency (formerly SHAPE Technical Centre), The Hague, The Netherlands, as a principal scientist. During this period, he was involved with various aspects of military SATCOM systems and represented NATO C3 Agency in various NATO committees and meetings. 
In 1993, he joined the Department of Electrical and Electronics Engineering of Eastern Mediterranean University, North Cyprus, as a full professor and was the Chairman from October 1994 to March 1996. Since March 1996, he is with the Department of Electrical and Electronics Engineering of Hacettepe University, Ankara, Turkey, where he acted as the Department Chairman during 1998-2001. He is currently the Head of the Telecommunications Group.

He conducted and supervised projects, served as a consultant and organized courses for various companies and institutions on diverse civilian and military communication systems. He served as a member of the executive committee of TUBITAK (Turkish Scientific and Technical Research Council)'s group on electrical and electronics engineering and informatics. He acted as reviewer in various national and EU projects and for distinguished journals. He was involved in the technical programme committee of many national and international conferences. He served as the Chair of $19^{\text {th }}$ IEEE Conference on Signal Processing and Communications Applications (SIU 2011). He represented Turkey to COST Action 262 on Spread Spectrum Systems and Techniques in Wired and Wireless Communications. He acted as the chairman of the COST Action 289 Spectrum and Power Efficient Broadband Communications.

He was involved with high frequency asymptotic techniques, reflector antennas, wave propagation in disturbed SATCOM links, design and analysis of military SATCOM systems and spread spectrum communications. His recent research interests include multi-carrier communications, channel modelling, cooperative communications, cognitive radio and MIMO systems. 
